\title{
Avaliação da Qualidade dos Registos de Medicação Prolongada: Estudo QMP
}

\author{
Quality Assessment of Long-Term Prescription Records: QMP Study
}

\author{
Ana AZEVEDO ${ }^{1}$, Brenda DOMINGUES ${ }^{2}$, Raquel PIMENTA DA ROCHA ${ }^{3}$, Rita EIRIZ ${ }^{4}$, Vanessa XAVIER² \\ Acta Med Port 2014 Sep-Oct;27(5):561-567
}

RESUMO

Introdução: A preocupação com a segurança na saúde torna essencial que todos os utentes, em particular os idosos por serem maioritariamente polimedicados, possuam uma lista completa e atualizada da sua medicação. Assim, propusemo-nos avaliar a qualidade dos registos eletrónicos de medicação prolongada.

Material e Métodos: Estudo observacional, transversal e descritivo, com componente analítico, no qual avaliámos a qualidade técnica dos registos de medicação prolongada nos idosos de quatro unidades de cuidados de saúde primários da região norte, antes e após uma intervenção. Nesta, efetuámos formação de boas práticas de registo aos médicos e promovemos a utilização do guia de Medicação Prolongada aos profissionais e utentes.

Resultados: Avaliámos 388 registos de 33 médicos. A categoria ideal, 'Medicação apropriada e posologia presente', aumentou de $23,5 \%$ para $48 \%(p<0,001)$. As restantes categorias 'Medicação inapropriada' e 'Medicação apropriada e posologia ausente' diminuíram de $16,7 \%$ para $7 \%(p=0,006)$ e de $59,8 \%$ para $46,0 \%(p=0,02)$, respetivamente. As variáveis condição de orientador de formação, local de trabalho, anos de Medicina Geral e Familiar e percentagem de idosos, apresentaram diferenças com significado estatístico no início do estudo, no entanto após a 'intervenção', apenas a percentagem de idosos continuou a apresentá-las.

Discussão: Neste estudo, os médicos aderiram à proposta de mudança, independentemente da idade, género, condição de orientador de formação, local de trabalho e anos de Medicina Geral e Familiar. Consultas mais prolongadas na população idosa poderão ter impedido alcançar a melhor categoria de registo.

Conclusão: Este trabalho original mostra que é necessário implementar medidas periódicas de formação pós-graduada para manutenção de registos médicos atualizados.

Palavras-chave: Cuidados de Longa Duração; Prescrição Electrónica; Prescrição de Medicamentos; Polimedicação.

\section{ABSTRACT}

Introduction: The growing concern surrounding health safety issues makes it essential that everyone, in particular the elderly due to their commonly prescribed multiple drugs, has a complete and up to date list of prescriptions. We planned to assess the quality of the electronic records of prolonged medication.

Material and Methods: This is an observational, transversal and descriptive study, with an analytical component, in which we assessed the technical quality of prolonged medication records of elderly patients of four primary health care, before and after a guided intervention. The doctors received training in good practice recording methods and both professionals and patients were stimulated to use the prolonged medication guide.

Results: We evaluated 388 medical records of 33 physicians. The ideal category 'Appropriated medication with posology' improved from $23.5 \%$ to $48 \%(p<0,001)$. The remaining categories 'Inappropriated Medication' and 'Appropriated medication but absent posology' decreased from $16.7 \%$ to $7 \%(p=0,006)$ and from $59.8 \%$ to $46.0 \%(p=0,02)$, respectively. The variables mentor's training skills, workplace, length of family practice and the percentage of elderly in the physician's list showed statistical significance differences at the beginning of the study which disappeared after the intervention, except for the latter.

Discussion: In this study, physicians accepted the proposed changes, regardless of age, gender, mentor's training skills, workplace or length of family practice. Longer duration appointments in the eldery group may be an obstacle in achieving the best results.

Conclusion: This original study reveals the necessity to implement periodic postgraduate training to encourage physicians to keep medical records up to date.

Keywords: Long-term Care; Electronic Prescribing; Drug Prescriptions; Family Practice; Aged.

\section{INTRODUÇÃO}

Tendo em conta o atual funcionamento do Serviço Nacional de Saúde (SNS) Português onde há livre circulação do doente e consequente multiplicidade de prescritores, sendo possível pedir a renovação da medicação prolongada pela Internet ${ }^{1}$ e onde se prevê a possibilidade de consultar o processo eletrónico de um doente em qualquer unidade de saúde do SNS, ${ }^{2}$ parece essencial um registo eletrónico da medicação prolongada permanentemente atualizado.
Apesar de não terem sido encontradas referências bibliográficas nesse sentido, a nossa experiência pessoal indica que esta não é a realidade do dia-a-dia.

Após uma revisão da bibliografia disponível, verificámos a existência de vários estudos relacionados com a polimedicação, sobretudo nos idosos, e em relação à vantagem do uso de sistemas eletrónicos no apoio à decisão médica. ${ }^{3-10}$ No entanto, não encontrámos estudos referentes à

1. Unidade de Saúde Familiar Sete Caminhos. Agrupamentos de Centros de Saúde de Gondomar. Gondomar. Portugal.

2. Unidade de Cuidados de Saúde Primários de Vale Formoso. Agrupamentos de Centros de Saúde do Porto Oriental. Porto. Portugal.

3. Unidade de Saúde Familiar Faria Guimarães. Agrupamentos de Centros de Saúde do Porto Oriental. Porto. Portugal.

4. Unidade de Saúde Familiar de São João do Porto. Agrupamentos de Centros de Saúde Porto Ocidental. Porto. Portugal.

Recebido: 12 de Dezembro de 2013 - Aceite: 10 de Fevereiro de 2014 | Copyright $\odot$ Ordem dos Médicos 2014 
qualidade dos registos de medicação prolongada.

A evolução da ciência na saúde proporcionou um aumento da esperança média de vida, em parte devido à prevenção e tratamento de patologias outrora fatais. Este fenómeno originou um aumento da prevalência das doenças crónicas e do número de indivíduos polimedicados, realidade mais evidente na população idosa. ${ }^{3-8,}$ 11-18

A adesão à terapêutica é menor em indivíduos portadores de doenças crónicas devido à necessidade de medicação prolongada ${ }^{19} \mathrm{e}$ também naqueles com esquemas terapêuticos complexos, isto é, com múltiplos fármacos ou tomas. ${ }^{3,7,11}$ Este tipo de esquemas não só diminui a adesão à terapêutica, como também aumenta o risco de erros de medicação; risco todavia maior quando associado às alterações fisiológicas do envelhecimento. ${ }^{4-6,11-13,15,19}$

Os sistemas eletrónicos de informação e decisão terapêutica e a informação escrita são fatores que contribuem para minimizar os erros de prescrição e favorecem a adesão à medicação, melhorando a prestação de cuidados e contribuindo para a redução de custos. ${ }^{3-10}$

Por tudo o que foi referido, surgiu a necessidade de conhecer a realidade portuguesa em relação ao registo da medicação prolongada, para que se possam implementar medidas para melhorar a prestação de cuidados, minimizar os erros de prescrição e os custos nos cuidados de saúde.

Neste sentido realizámos um estudo com o objetivo de avaliar, garantir e melhorar a qualidade técnica dos registos médicos eletrónicos de medicação prolongada, doravante designada Medicação Prolongada, numa amostra de utentes idosos pertencentes às listas dos médicos de família de quatro unidades de saúde da região norte, de forma a prevenir erros e otimizar os recursos disponíveis. Adicionalmente, determinámos a relação entre os resultados obtidos e as características dos médicos em análise.

\section{MATERIAL E MÉTODOS}

Estudo observacional, transversal e descritivo, com componente analítico, no qual avaliámos a Medicação Prolongada numa amostra representativa e aleatória de utentes idosos das listas de todos os médicos de quatro unidades de cuidados de saúde primários da região norte $(n=33)$. As quatro unidades incluídas foram: Unidade de Cuidados de Saúde Personalizados (UCSP) Vale Formoso, Unidade de Saúde Familiar (USF) Faria Guimarães, USF São João do Porto e USF Sete Caminhos. Este estudo decorreu entre março de 2012 e junho de 2013 e compreendeu três fases: avaliação, intervenção e reavaliação.

As fontes de dados utilizadas foram o Módulo de Informação e Monitorização das Unidades Funcionais ${ }^{\circledR}$ para as listas de utentes, e o Sistema de Apoio ao Médico ${ }^{\circledR}\left(\mathrm{SAM}^{\circledR}\right)$ para os registos clínicos. As características dos médicos foram obtidas por método de entrevista, utilizando um questionário elaborado por nós.

Na fase de avaliação, caracterizámos a população médica em relação à Idade, Género, Anos de Medicina Geral e Familiar (MGF), Local de Trabalho, Percentagem (\%) de Idosos na lista de utentes e condição de Orientador de For- mação (variáveis independentes).

$\mathrm{Na}$ fase de avaliação e de reavaliação, para podermos avaliar os registos de Medicação Prolongada (variável dependente), calculámos uma amostra proporcional e randomizada de utentes com idade igual ou superior a 65 anos e inscritos nas listas dos médicos. Utilizámos um intervalo de confiança de $95 \%$ e assumimos uma prevalência de 50\% por ausência de referências bibliográficas com um erro máximo $\beta$ de $5 \%$, obtendo uma amostra de 388 idosos.

Por falta de consenso nas referências bibliográficas revistas, e para efeitos deste estudo, a Medicação Prolongada foi definida por nós como a existência de pelo menos uma prescrição renovável de fármacos do tipo 'Tratamento prolongado', ou a existência de pelo menos três prescrições de fármacos do tipo 'Tratamento de curta ou de média duração', nos últimos 12 meses, no item correspondente do programa informático $\mathrm{SAM}^{\circledR} .{ }^{20,21}$

Categorizámos o registo de Medicação Prolongada em:

Categoria 0 - Medicação inapropriada: ausência de fármacos no item 'medicação prolongada' do $\mathrm{SAM}^{\circledR}$, na presença de histórico de prescrição de acordo com os critérios acima referidos.

Categoria 1 - Medicação apropriada e posologia ausente: existência de um ou mais fármacos no item 'medicação prolongada' do $\mathrm{SAM}^{\circledR}$ e ausência do número de unidades e/ou sua frequência de administração, em pelo menos um dos fármacos.

Categoria 2 - Medicação apropriada e posologia presente: existência de um ou mais fármacos no item 'medicação prolongada' do $\mathrm{SAM}^{\circledR} \mathrm{com}$ a descrição do número de unidades e sua frequência de administração, em todos os fármacos; ou a ausência de fármacos no item 'medicação prolongada' do $\mathrm{SAM}^{\circledR}$ em utentes sem medicação que cumpram os critérios acima definidos.

Os critérios de exclusão que aplicámos foram: ausência de consentimento do médico de família para participar no estudo; ausência do médico durante a intervenção geral e impossibilidade de realização de uma intervenção individual; ausência prolongada (igual ou superior a um mês) do médico incluído no estudo entre a intervenção e a reavaliação; utente falecido ou em processo de transferência para outra unidade de saúde. Em caso de exclusão de um utente foi incluído o seguinte da lista de seriação.

$\mathrm{Na}$ fase de intervenção, apresentámos os resultados obtidos na avaliação em reunião de serviço multidisciplinar de cada unidade de saúde. Aos médicos realizámos uma formação com vista a melhorar a qualidade dos registos através de uma apresentação audiovisual a explicitar as boas práticas de registo Medicação Prolongada. Aos enfermeiros e secretários clínicos solicitámos a sinalização de utentes sem guia de medicação prolongada bem como a necessidade de apresentação da mesma aquando do contacto para renovação de receituário. Alertámos os utentes para a importância de serem portadores da guia de medicação prolongada atualizada através da distribuição de panfletos e afixação de cartaz informativo em cada unidade de saúde. 
Na fase de reavaliação, realizada dez meses após a intervenção, avaliámos e comparámos apenas os resultados de Medicação Prolongada, após cálculo de nova amostra segundo os critérios já descritos.

O presente estudo foi aprovado pela Comissão de Ética para a Saúde da Administração Regional de Saúde do Norte.

$\mathrm{Na}$ análise estatística descrevemos as variáveis categóricas através de frequências absolutas e relativas, e as variáveis contínuas através da média e do desvio padrão ou da mediana e percentis, em função da simetria da sua distribuição. Para testar hipóteses sobre a distribuição de variáveis contínuas com distribuição não normal, utilizámos os testes não paramétricos de Mann-Whitney e de Kruskal-Wallis, em função da natureza das hipóteses testadas. Para testar hipóteses sobre a independência de variáveis categóricas aplicámos o teste de Qui-quadrado de independência, o teste exato de Fisher ou a correlação de Spearman, conforme apropriado. Efetuámos esta análise estatística utilizando o Statistical Package for the Social Sciences versão 18.0 para Windows ${ }^{\circledR}$.

Adotámos o nível de significância estatística de 0,05.

\section{RESULTADOS}

Dos 33 médicos incluídos, 55\% eram do género masculino, com idades entre os 37 e os 61 anos (média de 53 anos), exercendo a especialidade em média há 23 anos. Apenas 33\% dos médicos eram orientadores de formação e a percentagem média de idosos nas listas era de $19 \%$ (Tabela 1).

$\mathrm{Na}$ fase de avaliação obtivemos para as categorias 'Medicação inapropriada', 'Medicação apropriada e posologia ausente' e 'Medicação apropriada e posologia presente' valores de 16,8\% ( $n=65$, mediana = 1), 59,8\% ( $n=232$, mediana $=7)$ e $23,5 \%(n=91$, mediana $=3)$ respetivamente. Na reavaliação estes foram de 7,0\% $(n=26$, mediana $=0)$, $46,0 \%(n=177$, mediana $=5)$ e 48,0\% ( $n=185$, mediana $=8$ ) respetivamente (Fig. 1). Ao compararmos os registos entre as fases de avaliação e reavaliação, observámos um aumento da categoria Medicação apropriada e posologia presente, associada a uma diminuição nas outras duas categorias; esta melhoria foi estatisticamente significativa $(p<$ 0,001) (Fig. 1).

Comparámos a influência das características dos médicos nos registos de Medicação Prolongada nas fases de avaliação e reavaliação (Tabelas 2 e 3). Nas variáveis Género, Idade e Anos de MGF, não verificámos diferenças significativas, isto é, estas não parecem ter influenciado os resultados, em ambas as fases. Já para as variáveis Local de Trabalho, Orientador de Formação e Percentagem de Idosos encontrámos diferenças com significado estatístico. $\mathrm{Na}$ variável Local de Trabalho observámos que na fase de avaliação, os médicos da UCSP apresentavam mais registos de Medicação inapropriada $(p=0,008)$, no entanto, na reavaliação esta diferença deixou de existir (Tabela 2).

Verificámos que os Orientadores de Formação apresentavam melhores registos inicialmente ao terem menos
Tabela 1 - Descrição das variáveis independentes

\begin{tabular}{|ll}
\hline Género & $15(45 \%)$ \\
\hline Feminino & $18(55 \%)$ \\
\hline Masculino & $22(67 \%)$ \\
\hline Orientador de Formação & \\
\hline Não & $11(33 \%)$ \\
\hline Sim & \\
\hline Local de Trabalho & $26(79 \%)$ \\
\hline USF & $7(21 \%)$ \\
\hline UCSP & \\
\hline Idade (anos) & $53 \pm 6(37-61)$ \\
\hline Média & 51 \\
\hline P25 & 56 \\
\hline Mediana & 57 \\
\hline P75 & 29 \\
\hline Anos de MGF & 23 \\
\hline Média & $29(2-35)$ \\
\hline P25 & \\
\hline Mediana & \\
\hline P75 & \\
\hline
\end{tabular}

\section{Percentagem de Idosos}

$\begin{array}{ll}\text { Média } & 18,8 \pm 3,63 \\ & (11,9 \%-25,4 \%) \\ \text { P25 } & 16,25\end{array}$

Mediana $\quad 18,31$

$\begin{array}{ll}\text { P75 21,32 } & \end{array}$

registos de Medicação inapropriada $(p=0,011)$. Após a intervenção, também esta diferença deixou de se verificar, demonstrando uma melhoria do registo por parte de todos os médicos, incluindo os médicos não orientadores, para todas as categorias de registo (Tabela 2).

No que respeita à variável Percentagem de Idosos na fase de avaliação, obtivemos uma correlação positiva para a categoria 0 , isto é, quanto maior a sua percentagem, maior o número de registos com Medicação inapropriada $(p=0,043)$. Na fase de reavaliação, esta correlação positiva verificou-se para a Medicação apropriada e posologia ausente ( $p=0,007)$ (Tabela 3).

Para avaliarmos a tendência dos registos médicos após a formação, efetuámos uma análise comparativa médico-a-médico nos dois momentos temporais, com categorização da mesma em 'manteve/melhorou' ou 'piorou' (Tabela 4).

De uma forma global, o registo na Medicação inapropriada manteve/melhorou, devido a uma diminuição dos registos incorretos (Tabela 4). Na categoria Medicação apropriada e posologia ausente houve um aumento do número de registos (Tabela 4). Este fenómeno corresponde a uma 


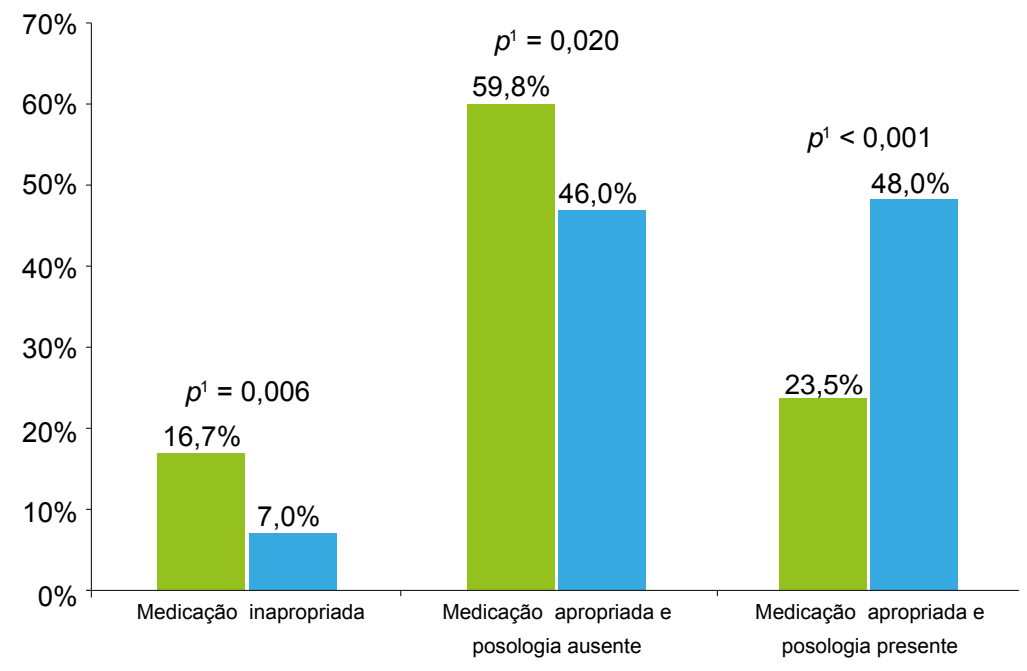

Avaliação

Reavaliação

Figura 1 - Comparação dos registos antes e após a intervenção

$p^{1}$ : Teste de Wilcoxon.

Tabela 2 - Comparação dos registos, por variável independente dicotómica, antes e após intervenção

\section{Categorias de Registo}

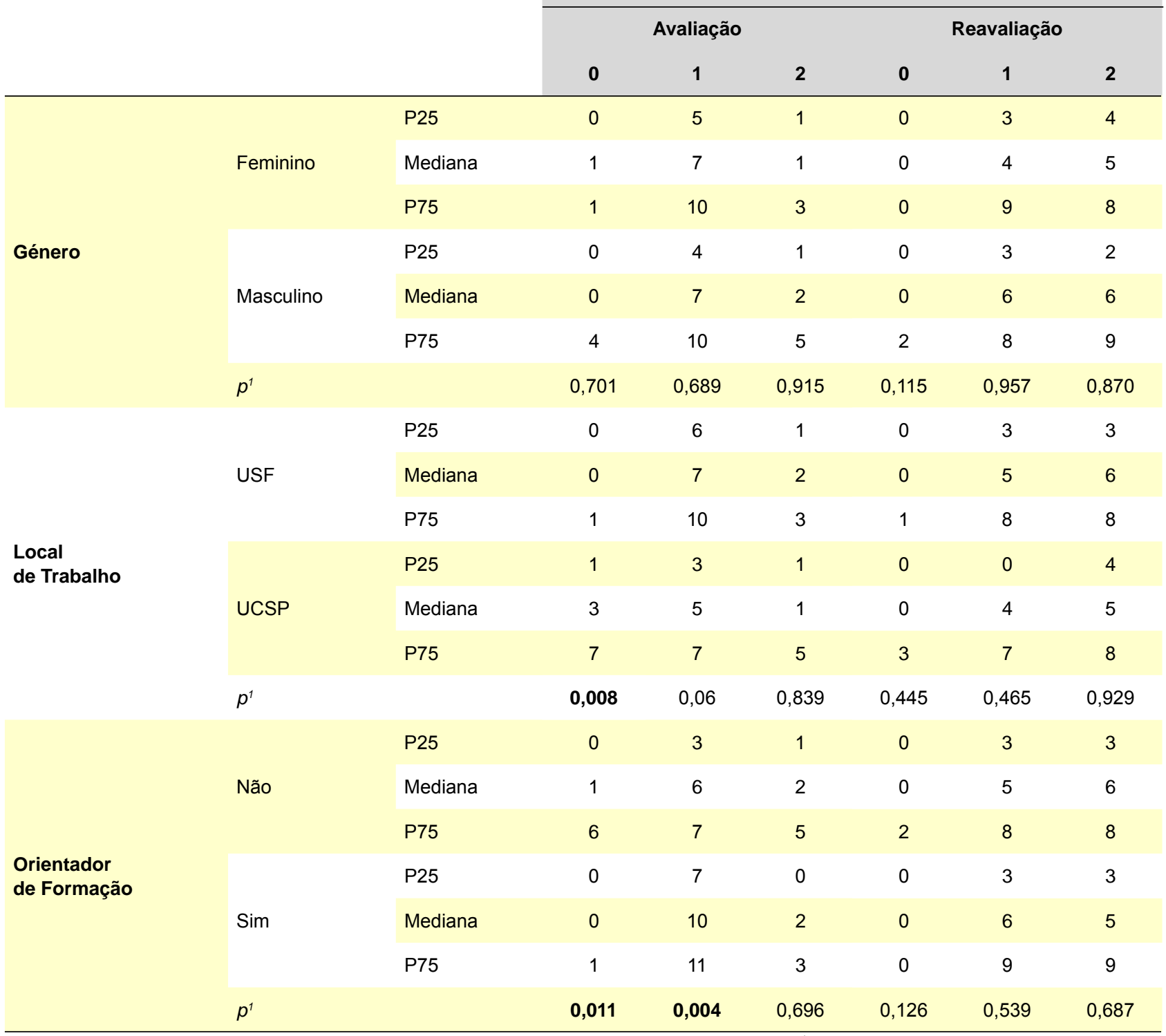

0- Medicação inapropriada; 1- Medicação apropriada e posologia ausente; 2 - Medicação apropriada e posologia presente; $p^{1}$ - Teste de Mann Whitney. 
Tabela 3 - Comparação dos registos, por variável independente continua, antes e após a intervenção

\begin{tabular}{|c|c|c|c|c|c|c|c|}
\hline & & \multirow{2}{*}{\multicolumn{6}{|c|}{ Categorias de Registo }} \\
\hline & & & & & & & \\
\hline & & \multicolumn{3}{|c|}{ Avaliação } & \multicolumn{3}{|c|}{ Reavaliação } \\
\hline & & 0 & 1 & 2 & 0 & 1 & 2 \\
\hline \multirow{2}{*}{ Idade } & Coeficiente de correlação & 0,210 & 0,042 & $-0,080$ & 0,255 & 0,009 & $-0,032$ \\
\hline & $p^{1}$ & 0,241 & 0,815 & 0,659 & 0,152 & 0,961 & 0,859 \\
\hline \multirow[b]{2}{*}{ Anos de MGF } & Coeficiente de correlação & 0,261 & 0,099 & $-0,092$ & 0,172 & $-0,062$ & 0,142 \\
\hline & $p^{1}$ & 0,142 & 0,582 & 0,611 & 0,339 & 0,732 & 0,431 \\
\hline \multirow{3}{*}{$\%$ Idosos na Lista } & Coeficiente de correlação & 0,355 & $-0,043$ & 0,268 & 0,284 & 0,046 & $-0,051$ \\
\hline & & & & & & & \\
\hline & $p^{1}$ & 0,043 & 0,812 & 0,132 & 0,109 & 0,007 & 0,779 \\
\hline
\end{tabular}

Tabela 4 - Efeito global da intervenção por categoria

Efeito da intervenção por categoria

0

Manteve/ melhorou

19 (58\%)
Piorou Manteve/ melhorou
1

2
Piorou

$4(12 \%)$

0- Medicação inapropriada; 1- Medicação apropriada e posologia ausente; 2 - Medicação apropriada e posologia presente.

melhoria devido à passagem de registos da Medicação inapropriada para a categoria seguinte. No entanto, não podemos excluir que pontualmente não tenham ocorrido casos de registos totalmente corretos na fase de avaliação que tenham piorado posteriormente. Na categoria Medicação apropriada e posologia presente, constatámos que $88 \%$ dos médicos manteve/melhorou os seus registos (Tabela 4).

\section{DISCUSSÃo}

Esperávamos que os médicos mais velhos, por eventualmente terem uma menor facilidade no registo eletrónico, tivessem piores resultados, contudo, tal não se verificou, o que demonstra a adesão dos médicos às novas tecnologias. Também para a variável Género não observámos diferenças significativas nos registos.

Em relação aos médicos orientadores de formação, acreditávamos que tivessem melhores registos na avaliação pela questão didática, o que se verificou. Na reavaliação, esperávamos que neste grupo por haver médicos internos, a sensibilização para a melhoria dos registos fosse maior, e que tal proporcionasse diferenças, o que não ocorreu. De qualquer forma, o facto de internos de formação específica, em particular, nós na qualidade de autoras, terem participado ativamente no estudo, em nome dos orientadores de formação, pode constituir um viés nos registos dos respetivos médicos, ainda que não se tenham verificado diferenças estatisticamente significativas.

Relativamente ao Local de Trabalho, esperávamos que os registos fossem melhores nas USF, uma vez que os médicos destas instituições estão mais habituados a cumprir indicadores, podendo estar mais recetivos a modificações da sua prática diária. Apesar de na avaliação o panorama ser pior na UCSP, as diferenças foram corrigidas após a intervenção, o que demonstra que o Local de Trabalho não influenciou a atitude dos médicos para a mudança.

Quanto aos Anos de MGF, prevíamos que os médicos que fizeram o internato mais recentemente, em especial após a existência de registos informatizados, tivessem mais facilidade para preencher os registos corretamente. $\mathrm{Na}$ avaliação, para a categoria Medicação inapropriada, os especialistas mais recentes tiveram melhores registos globais. Após a intervenção as diferenças diluíram-se, mostrando que os Anos de MGF não influenciaram a adaptação dos médicos.

No que respeita à Percentagem de Idosos, verificámos que esta foi diretamente proporcional ao número de registos totalmente incorretos na fase de avaliação. Este fenómeno poderá ser explicado pelo facto de a população idosa apresentar múltiplas patologias, o que prolonga o tempo da consulta e deixa menos espaço para a atualização dos registos. Na reavaliação, verificou-se uma melhoria dos registos passando a correlação a ser positiva com a categoria Medicação presente e posologia ausente. Mais uma vez, especulámos se a melhoria não foi superior por estas serem consultas habitualmente mais prolongadas neste grupo etário. Estes dados permitem abrir a discussão para qual será a Percentagem de Idosos adequada numa lista 
de utentes de um médico de família, de forma a otimizar tanto os custos quanto os ganhos em saúde.

Apesar de a população idosa provavelmente não ser representativa de uma lista de utentes, foi nossa opção estudá-la por ser habitualmente polimedicada e com múltiplos contatos com as unidades de saúde, o que proporciona uma maior oportunidade de atualização da Medicação Prolongada num menor espaço de tempo. Por este motivo esperávamos que uma elevada Percentagem de Idosos na lista apresentasse um melhor registo após a intervenção. Contudo, verificou-se que isto não é necessariamente verdade, podendo ser explicado pelo trabalho mais exaustivo que esta população exige.

Um ponto fraco/ limitação importante do estudo é o facto de ser um estudo local, com inclusão de apenas quatro instituições de saúde. Além disso, a pequena amostra de médicos (33) poderá constituir um viés para alguns dos resultados encontrados, justificando tanto que algumas das diferenças esperadas não se tenham verificado como o oposto. Estes fatores dificultam assim a extrapolação/generalização dos resultados.

Da mesma maneira, considerando que não foi analisado o efeito dos outros grupos profissionais e utentes sobre os resultados, isto pode constituir um viés do nosso trabaIho.

São pontos fortes do nosso estudo, a inovação, pela sua tipologia de Melhoria Contínua da Qualidade com uma vertente de investigação associada, considerando a atualidade do tema, uma vez que cada vez mais as unidades de saúde são alvo de auditorias ou processos de acreditação. Também inova por abordar uma área muito importante na saúde e ainda não parametrizada por indicadores ou auditorias, como é o registo da Medicação Prolongada, com prováveis ganhos em saúde e melhor gestão dos recursos económicos existentes. Termos obtido a participação da totalidade de médicos das unidades de saúde em estudo e não terem existido perdas ao longo do mesmo, bem como ter sido realizado no âmbito dos cuidados de saúde primários (área habitualmente com menor visibilidade nos meios científicos), são pontos a favor desta investigação. Por últi-

\section{REFERÊNCIAS}

1. Ministério da Saúde. Manual do eAgenda. Administração Central do Sistema de Saúde. [Internet]. [acedido em 2011 Abr 14]. Disponível em http://www.acss.min-saude.pt/Portals/0/Circulares/eAgendamanual.pdf.

2. Serviços Partilhados do Ministério da Saúde, EPE. PDS-Plataforma de Dados da Saúde. [Internet]. [Consultado 2013 Nov 6]. Disponível em http://spms.min-saude.pt/2013/11/pds-plataforma-de-dados-da-saude/.

3. Cima CF, Freitas RS, Lamas MC, Mendes CA, Neves AC, Fonseca C. Consumo de medicação crónica: avaliação da prevalência no norte de Portugal. Rev Port Clin Geral. 2011;27:20-7.

4. Sousa S, Pires A, Conceição C, Nascimento T, Grenha A, Braz L. Polimedicação em doentes idosos: adesão à terapêutica. Rev Port Clin Geral. 2011;27:176-82.

5. Tamblyn R, Huang A, Perreault R, Jacques A, Roy D, Hanley J, et al. The medical office of the 21st century (MOXXI): effectiveness of computerized decision-making support in reducing inappropriate prescribing in primary care. CMAJ. 2003;169:549-56.

6. Pham CB, Dickman RL. Minimizing adverse drug events in older patients. Am Fam Physician. 2007;76:1837-44.

7. Ghandi TK, Weingart SN, Seger AC, Borus J, Burdick E, Poon EG, et mo, de referir a inclusão de diferentes grupos profissionais e utentes neste estudo, apesar de a análise ter sido efetuada apenas para os registos médicos.

De salientar que a inclusão de todos os médicos das quatro unidades demonstra a disponibilidade da classe para melhorar os seus registos, com as vantagens económicas e ganhos em saúde de daqui se obtêm.

\section{CONCLUSÃO}

Com este estudo verificámos que os registos de Medicação Prolongada nem sempre existem, ou quando existem nem sempre estão completos. Isto pode dever-se ao facto de não existirem, até à data da realização deste trabaIho, indicadores ou auditorias a este parâmetro. Contudo, constatámos que estes podem ser melhorados através de intervenções que sensibilizem os profissionais de saúde, em especial os médicos, e os utentes para a necessidade de mudança, com prováveis ganhos em saúde.

No entanto, são necessários mais estudos para possibilitar a generalização dos resultados, definir um padrão de qualidade e perceber qual a percentagem de idosos ideal na lista de um médico de família, de forma a permitir um registo adequado da Medicação Prolongada.

De igual forma, estudos confirmatórios destes dados poderão ser uma ajuda no sentido de se implementarem medidas periódicas de atualização pós-graduada para estimular os médicos ao longo da sua carreira a manterem um registo de Medicação Prolongada atualizado e completo.

\section{CONFLITOS DE INTERESSE}

As autoras declaram não existir conflitos de interesse.

\section{FONTES DE FINANCIAMENTO}

Este trabalho foi financiado na sua totalidade pelas autoras, não havendo lugar a subsídios ou bolsas.

Parte dos resultados foram apresentados no $18^{\circ}$ Congresso Nacional de Medicina Geral e Familiar na Covilhã a 29 de Setembro de 2013, tendo recebido o prémio de melhor comunicação na categoria de 'Melhoria Contínua da Qualidade'.

al. Outpatient prescribing errors and the impact of computerized prescribing. J Gen Intern Med. 2005;20:837-41.

8. Sanguansak T, Morley MG, Yospaiboon Y, Lorch A, Hedt B, Morley $\mathrm{K}$. The impact of preprinted prescription forms on medication prescribing errors in an ophthalmology clinic in northeast Thailand: a non-randomised interventional study. BMJ Open. 2012;2:e000539.

9. van Doormaal, van den Bemt PM, Zaal RJ, Egberts AC, Lenderink BW, Kosterink JG, et al. The influence that electronic prescribing has on medication errors and preventable adverse drug events: an interrupted time-series study. J Am Med Inform Assoc. 2009;16:816-25.

10. Devine EB, Hansen RN, Wilson-Norton JL, Lawless NM, Fisk AW, Blough DK, et al. The impact of computerized provider order entry on medication errors in a multispecialty group practice. J Am Med Inform Assoc. 2010;17:78-84.

11. Rocha $\mathrm{CH}$, de Oliveira AP, Ferreira C, Faggiani FT, Schroeter G, de Sousa $A C$, et al . Adesão à terapêutica médica em idosos de Porto Alegre, RS. Cien Saude Colet. 2008;13:703-10.

12. Brekke M, Rognstad S, Straand J, Furu K, Gjelstad S, Bjørner T, et al. Pharmacologically inappropriate prescriptions for elderly patients in 
general practice: How common? Baseline data from The Prescription Peer Academic Detailing (Rx-PAD) study. Scand J Prim Health Care. 2008;26:80-5

13. Bregnhøj L, Thirstrup S, Kristensen MB, Bjerrum L, Sonne J. Combined intervention programme reduces inappropriate prescribing in elderly patients exposed to polypharmacy in primary care. Eur J Clin Pharmacol. 2009;65:199-207.

14. Nóbrega OT, Karnikowski M. A terapia medicamentosa no idoso: cuidados na medicação. Cien Saude Colet. 2005;10:309-13.

15. Hanlon JT, Weinberger M, Samsa GP, Schmader KE, Uttech KM, Lewis IK, et al. A randomized, controlled trial of a clinical pharmacist intervention to improve inappropriate prescribing in elderly outpatients with polypharmacy. Am J Med. 1996;100:428-37.

16. Ferner RE. An agenda for UK clinical pharmacology: medication errors. Br J Clin Pharmacol. 2012;73:912-6.
17. Fialová D, Onder G. Medication errors in elderly people: contributing factors and future perspectives. Br J Clin Pharmacol. 2009;67:641-5

18. Ryan C, O'Mahony D, Kennedy J, Weedle P, Byrne S. Potentially inappropriate prescribing in an Irish elderly population in primary care. $\mathrm{Br} \mathrm{J}$ Clin Pharmacol. 2009;68:936-47.

19. Osterberg L, Blaschke T. Adherence to medication. N Engl J Med. 2005;353:487-97.

20. Diário da República Eletrónico. Portaria n. ${ }^{\circ} 1471 / 2004$, de 21 de Dezembro. Diário da República-1 ${ }^{a}$ Série B. [Internet]. [Consultado $2012 \mathrm{Ab}$ 20]. Disponível em http://dre.pt/pdf1s/2004/12/297B00/72407243.pdf.

21. Autoridade Nacional do Medicamento e Produtos de Saúde, IP. Deliberação 173/CD/2011. Infarmed. [Internet]. [Consultado 2012 Abr 20]. Disponível em http://www.infarmed.pt/portal/page/portal/INFARMED/ LEGISLACAO/ACTOS_SUJEITOS_A_PUBLICACAO_NO_SITE_DO INFARMED/173_CD_2011.pdf. 


\section{Avaliação da Qualidade dos Registos de Medicação Prolongada: Estudo QMP}

Acta Med Port 2014:27:561-567

Publicado pela Acta Médica Portuguesa, a Revista Científica da Ordem dos Médicos

Av. Almirante Gago Coutinho, 151

1749-084 Lisboa, Portugal.

Tel: +351218428215

E-mail: submissao@actamedicaportuguesa.com

www.actamedicaportuguesa.com

ISSN:0870-399X | e-ISSN: 1646-0758

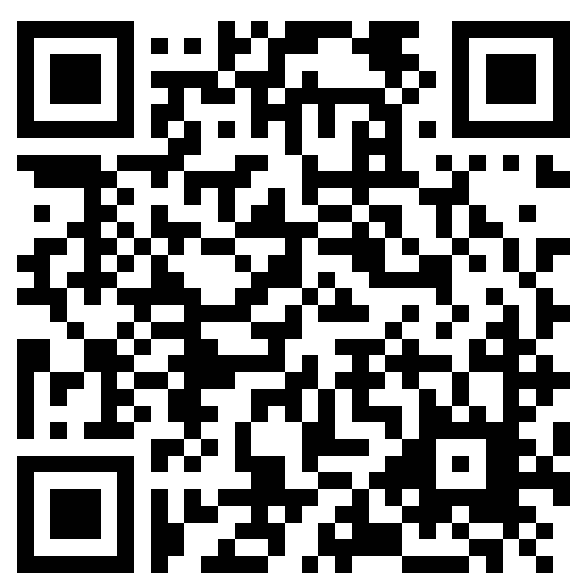

